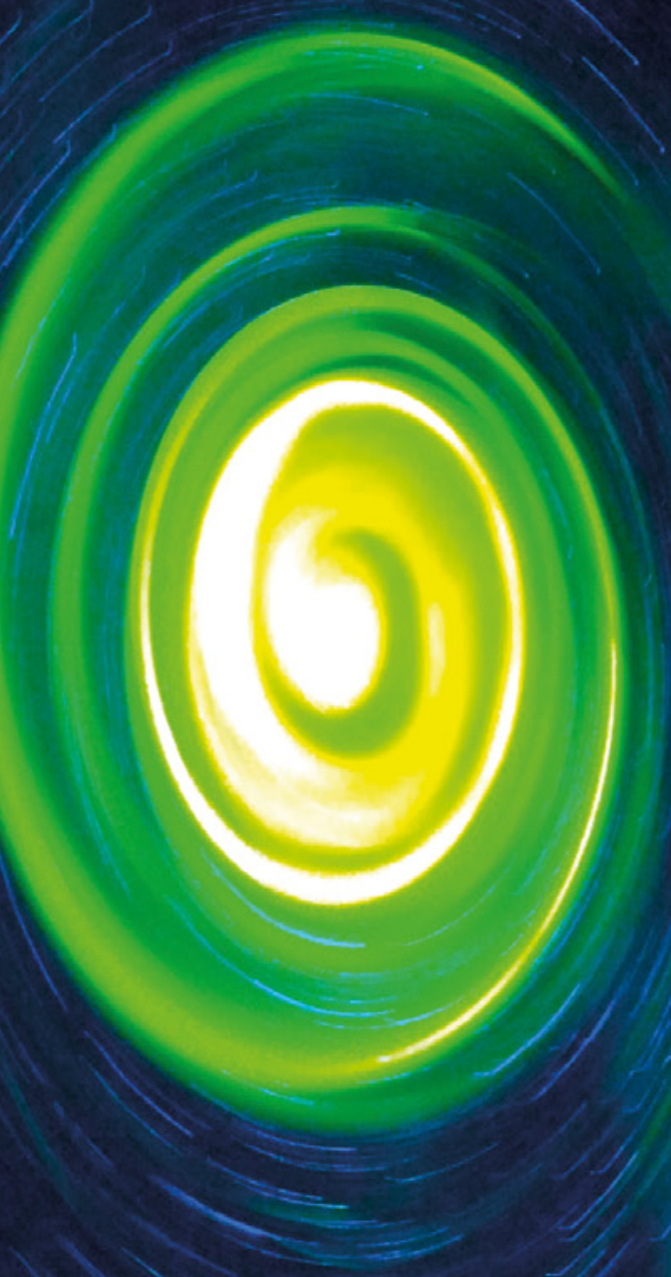

\title{
A brief history of the Coriolis force
}

- Theo Gerkema ${ }^{1}$ and Louis Gostiaux ${ }^{2}$ DOI: $10.1051 / \mathrm{epn} / 2012202$

1 NIOZ, Texel, Netherlands and ${ }^{2}$ LEGI, Grenoble, France

In 1835, Gustave Coriolis derived the expression of a force acting in rotating systems, now known as the Coriolis force. His work was inspired by rotating devices such as waterwheels. However, the one rotating device that has always been with us is the Earth itself. Indeed, the earliest studies on how moving objects behave in a rotating system were directed to the Earth's diurnal rotation. Here we trace the history of these studies, which started two centuries before Coriolis. 
T he first ideas concerning the influence of the Earth's diurnal rotation on terrestrial objects came with the debate on the very existence of that rotation. This was inevitable, for Aristotelian physics offered a seemingly weighty argument against it: everything lifted into the sky, such as birds or clouds, would no longer share the Earth's rotational movement and hence should drift away to the west at a gigantic speed. Since this is not what we observe, the conclusion had to be that the Earth does not rotate. To move beyond this Aristotelian argument, a different notion of inertia was needed. It was provided by Galilei who put forward the idea that objects persist in their horizontal movement, by which he meant that they would continue their circular trajectory [3]; thus, a stone thrown up in the air does not suddenly lose its rotational motion but keeps on moving along with the Earth.

\section{Deflection of freely falling bodies and projectiles}

In 1668, Giovanni Borelli, a member of the Accademia del Cimento in Florence, examined what this principle of inertia implies for objects that are dropped from a tower [7]. On the rotating Earth, the top of a tower describes a larger zonal circle than its foot, and thus must have a higher (circular) velocity to the east. Borelli supposed that an object falling from the top must retain this excess in eastward velocity and hence land slightly to the east of the tower's foot. He calculated the magnitude of this deflection and found, for a tower of a height of $71 \mathrm{~m}$ (240 feet $^{1}$ ) and placed at the equator, a value of $2 \mathrm{~cm}$. We will not dwell on the details of his reasoning; notwithstanding some errors and approximations, he found the right direction and the right order of magnitude. He concluded that the effect was too small to be measured, as the deflection is easily dwarfed by other perturbing effects.

More than a century would pass before significant progress was made on this problem. In 1803, Laplace and Gauss independently derived an expression for the eastward deflection of freely falling objects:

$$
d=2 / 3 \Omega \cos (\Phi) \sqrt{\left(2 h^{3} / g\right)}
$$

Here $\Omega$ is the Earth's angular velocity $\left(7.292 \times 10^{-5} \mathrm{rad} / \mathrm{s}\right.$, i.e., $2 \pi$ divided by the sidereal day: $23 \mathrm{~h} 56 \mathrm{~m} 4 \mathrm{~s}$ ) and $\Phi$ is latitude; $g$ is the acceleration of gravity and $h$ the height from which the object falls. During the same period, experiments were carried out in which experimentalists carefully attempted to eliminate all disturbing influences. The most famous is the one by Ferdinand Reich in a mine pit $158.5 \mathrm{~m}$ deep, in Freiberg, Saxony [6]. On a total of 106 experiments he found a mean eastward deflection of $2.8 \mathrm{~cm}$, which agrees with the theoretical value. Despite all the precautions he took, the spread in the values is conspicuous (see Figure 1); the balls sometimes even deflected to the west!
A related but more complicated problem is that of the deflection of bodies launched in an arbitrary direction. Riccioli in his Almagestum Novum (1651) argued that, if the Earth rotates, a projectile fired northward should deflect to the east: the projectile comes from a latitude whose zonal circle is larger than the one to which it goes; the accompanying excess in eastward velocity would be retained by the projectile, amounting to a deflection to the east ${ }^{2}$. By contrast, Riccioli (incorrectly) expected no deflection for eastwardly fired objects. His reasoning implies that a systematic difference between northwardly and eastwardly fired objects should have been detected; he took the absence of any such evidence as an argument against the Earth's rotation [5].

Once again, it was Laplace who put the problem on a solid mathematical footing; in the fourth volume of his Mécanique Céleste he derived the equations governing the deflection of projectiles. He showed, in particular, that a body launched vertically upward would land slightly westward.

\section{Laplace and the "Traditional Approximation"}

Laplace's work on the deflection of projectiles was a spin-off of his earlier treatise on tides, which was published in its definitive form in the first volume of his Mécanique Céleste [8]. For a hypothetical ocean covering the entire planet, he examined their modes of propagation. To do this, he first had to derive the equations of motion including the effects of the Earth's rotation. Adopting the usual geographical coordinates, he demonstrated that these equations contain four terms that represent a deflecting force due to the Earth's rotation:

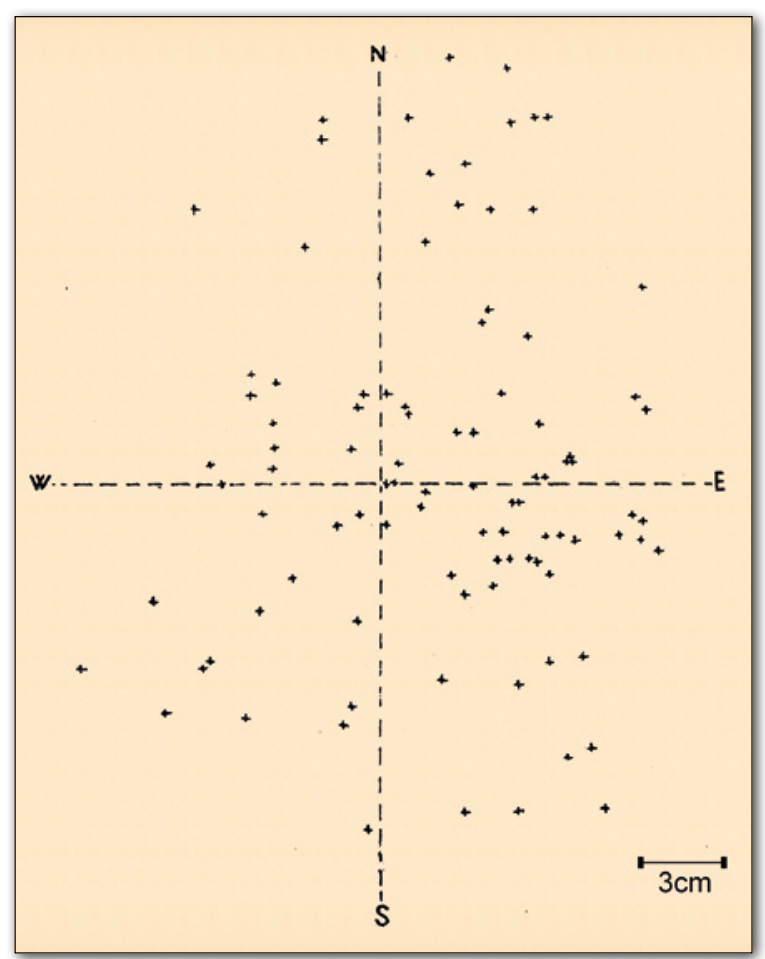

\begin{tabular}{l} 
NOTES \\
\hline Height of \\
the Torre \\
degli Asinelli \\
in Bologna, \\
from which \\
experiments \\
on freely falling \\
objects were \\
carried out at \\
the time. \\
\\
2 In fact, one \\
should apply \\
here the principle \\
of conservation \\
of angular \\
momentum; \\
this correction \\
doubles the \\
deflection, as was \\
first pointed out \\
by Brillouin [1].The \\
same applies to \\
Borelli's reasoning \\
on freely falling \\
bodies.
\end{tabular}

$\triangle$ FIG 1: The result of 106 experiments on freely falling balls in a minepit by Ferdinand Reich in 1831 (from [6]). 
moving bodies are subject to a deflecting force in a direction perpendicular to - and in magnitude proportional to - their velocity. Specifically, the effect of the four terms is as follows (see Figure 2):

- an eastward velocity $u$ induces (I) a southward force (whose expression is $-2 \Omega \sin (\Phi) u$ ) and (II) a vertically upward force $(2 \Omega \cos (\Phi) u)$;

- a northward velocity $v$ induces (III) an eastward force $(2 \Omega \sin (\Phi) v)$

- a vertically upward velocity $w$ induces (IV) a westward force $(-2 \Omega \cos (\Phi) w)$.

Forces are taken per unit of mass. These results apply to the Northern Hemisphere; in the Southern Hemisphere, $\Phi$ becomes negative, so that one should replace "southward" by "northward" for (I), and "eastward" by "westward" for (III).

His analysis contained yet another element that would prove influential. Laplace pointed out that the ocean is but a thin shell compared to the Earth's radius (the same is true for the atmosphere); this geometrical constraint means that large-scale motions must be quasi horizontal. This renders the terms (II) and (IV) relatively unimportant, for they involve vertical movements (either in velocity or in acceleration). Thus, Laplace neglects them and henceforth only takes into account the deflecting terms (I) and (III). Ever since, this approximation has been routinely made in studies on geophysical fluid dynamics and is aptly referred to as the "Traditional Approximation". In recent years, the validity of this approximation has

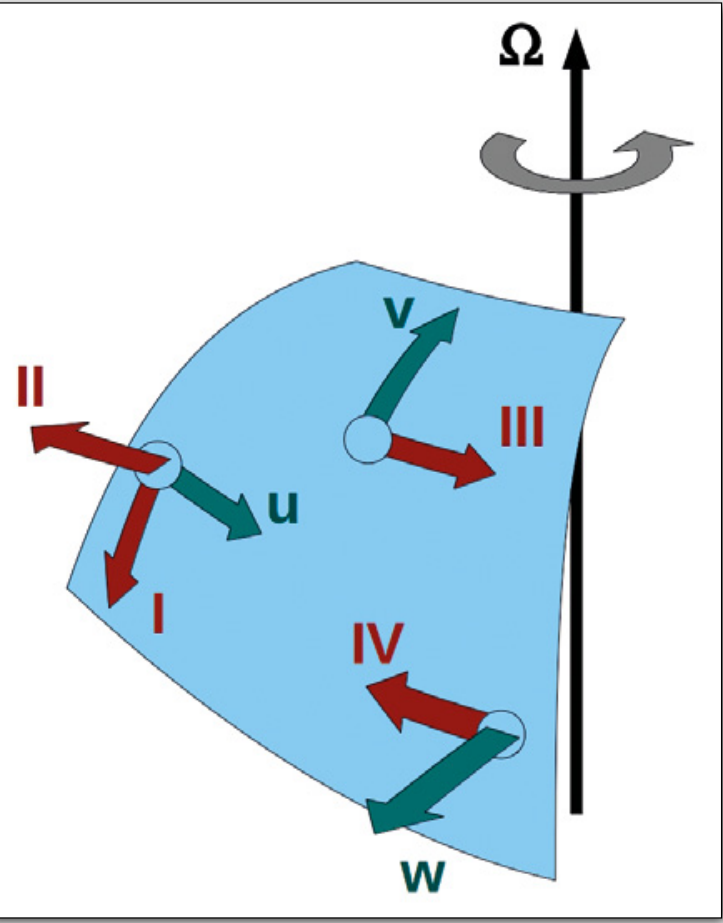

however come under renewed scrutiny for some types of geophysical flows [4].

Historically, (IV) was the first of the four effects to be probed into, for it is the term responsible for the eastward deflection of a freely falling body, the case studied by Borelli. By contrast, the effect of the other "non-traditional" term was the last to be identified. In the early $20^{\text {th }}$ century, when gravity measurements were carried out during seagoing scientific campaigns, it was found that its value was systematically smaller on an eastward moving ship, and larger on a westward moving one. (For example: for a ship going eastward at the equator at 13 knots, gravity $g$ is reduced by one-hundredth of a percentage point.) In 1919, Eötvös pointed out that this phenomenon is explained by term (II).

\section{Geostrophy}

In 1856, Ferrel adopted the terms (I) and (III) from Laplace's tidal theory and introduced them in meteorology [1]. In a qualitative way, the effect of term (III) had already been introduced by Hadley in an article on the Trade Winds in 1735, but term (I) was new in this field. Ferrel also clarified the way in which (I) causes a deflection: a particle of air which moves to the east is subject to an extra centrifugal force (since its own eastward velocity adds up to the already existing eastward velocity associated with the Earth's rotation). This extra force is oriented outward in the plane spanned by the zonal circle; it can be decomposed into a meridional and a radial component, yielding (I) and (II), respectively.

Ferrel constructed a schematic view of the general circulation of the atmosphere (Figure 3). In each hemisphere, he distinguished three zones (the trade winds near the equator, the westerlies at mid-latitudes, and the polar easterlies), which are separated by belts of low or high pressure (from equator to poles: the doldrums, subtropical high, subpolar low). Then, Ferrel assumed that the meridional gradient of pressure is balanced with term (I), featuring the zonal velocity. He thus introduced what we now know as the "geostrophic balance", according to which the winds follow the isobars rather than being perpendicular to them.

\section{Foucault's pendulum}

In 1851 Foucault carried out his famous experiment in which he observed a slow rotation of the vertical plane of the pendulum's oscillation. This effect is due to the terms (I) and (III); to a first approximation, the frequency of the plane's rotation is given by $\Omega \sin (\Phi)$. Soon after, many such experiments were carried out at different places by other experimentalists. They not only confirmed the original experiment but also testified to the great appeal a direct 
mechanical proof of the Earth's rotation had to many, even if few would by then have doubted its reality. There is an ironical twist here, for in fact the experiment had already been inadvertently done at a time when the Earth's rotation itself was still under debate. In notes from the 1660s, members of the Accademia del Cimento mention their experiments on pendulums and report a clockwise rotation of the vertical plane (Figure 4). They did not realize that the Earth's rotation may have something to do with it. For them, it was a nuisance; and afterwards they fixed the pendulum at two ropes to preclude this annoying perturbation!

\section{Coriolis}

The four terms (I) to (IV), first derived by Laplace, are none other than what we now know as the "Coriolis force". In 1835, Coriolis derived 'his' force in a theoretical treatise on the forces acting in rotating devices [2]. He called it the "force centrifuge composée"; the association with the name of Coriolis became common only by the late $19^{\text {th }}$ century. ${ }^{3}$ In fact, his paper past unnoticed in the first few decades after its publication. Notably, Poisson in his papers on the deflection of projectiles in 1837/1838 drew on Laplace's Mécanique Céleste and shows no awareness of Coriolis' paper. The same is true for early theoretical explanations of Foucault's pendulum (such as by Binet in 1851). Ferrel's work, too, is a case in point.

It is only natural that Laplace was much more influential than Coriolis. Firstly, because Laplace's work concerned the Earth's rotation, which, ever since Borelli, was the primary object of study in effects of deflection (the cases discussed above testify to that). By contrast, Coriolis' work was more abstract and the applications he envisaged were rooted in the industrial revolution, with rotating devices such as waterwheels - a wholly different context. Secondly, Laplace's derivation of the deflecting force preceded that by Coriolis by four decades. Historically, it would certainly be more accurate to speak of the "Laplace force". But, then, Laplace has already got so many things named after him, that it should be considered a matter of fairness to speak of the "Coriolis force"!

\section{About the authors}

Theo Gerkema is a researcher at the NIOZ Netherlands Institute for Sea Research on Texel, The Netherlands. His main areas of interest are internal waves in the ocean and coastal dynamics. Besides, he dabbles in the history of physics and oceanography.

Louis Gostiaux is a CNRS researcher at the LEGI, University of Grenoble, France. His topics of interest are oceanic internal waves and stratified fluid mechanics, mainly using laboratory and field observation.

\section{NOTE}

${ }^{3}$ E.g., Kamerlingh Onnes in his dissertation on Foucault's pendulum, in 1879

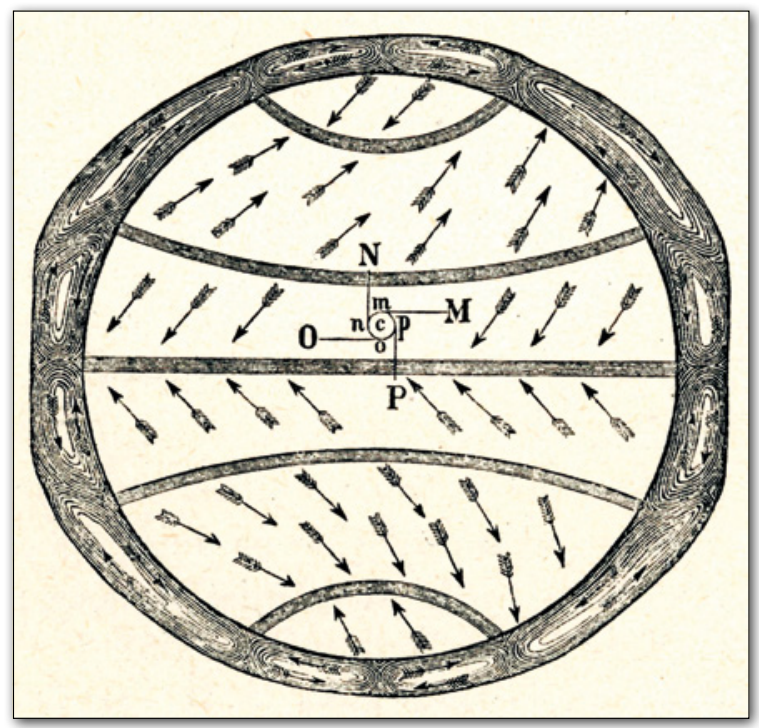

4 FIG 3:

Schematic view

of the general circulation of

the atmosphere, established by Ferrel in 1856 (from [1]). The flow pattern is a combination of meridional flows, associated with the three circulation cells in each hemisphere (depicted in the outer spherical shell); the easterlies and westerlies are explained by the zonal geostrophic balance.

\section{References}

[1] M. Brillouin, Mémoires originaux sur la circulation générale de l'atmosphère. Carré et Naud, Paris (1900).

[2] G. Coriolis, Sur les équations du mouvement relatif des systèmes de corps. J. Éc. Polytech. Paris, Cahier XXIV, Tome XV, 142 (1835).

[3] E.J. Dijksterhuis, The mechanization of the world picture. Oxford University Press (1961).

[4] T. Gerkema, J.T.F. Zimmerman, L.R.M. Maas and H. van Haren, Rev. Geophys. 46, 1 (2008). RG2004

[5] C.M. Graney, Physics Today 64 (8), 8 (2011).

[6] J.G. Hagen, La rotation de la terre, ses preuves mécaniques anciennes et nouvelles. Tipografia Poliglotta Vaticana, Roma (1911).

[7] A. Koyré, Trans. Am. Phil. Soc. 45 (4), 329 (1955).

[8] P.S. Laplace, Traité de Mécanique Céleste, Vol. 1. Crapelet, Paris (1798).

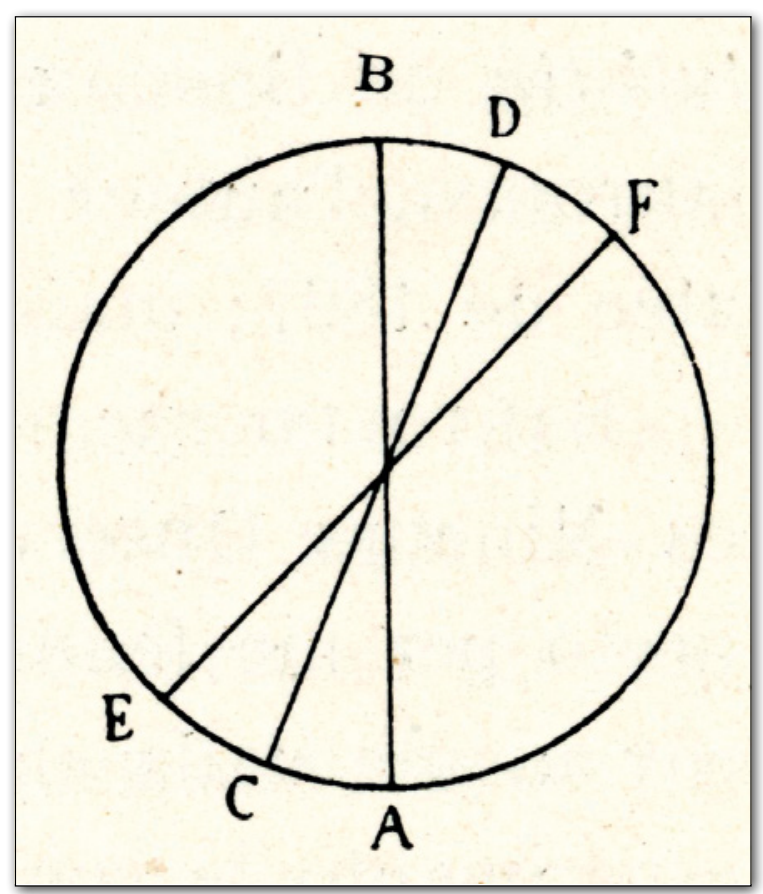

4 FIG 4: Foucault's pendulum avant la lettre. Extract from notes by Viviani (Accademia del (imento, 1660s): "We observed that all the pendulums at a single rope deviated from their plane [of oscillation] and always in the same sense, that is, according to the lines $A B, C D$, EF, etc" (Figure from [6]). 